\title{
A Note on a Characterization of Gompertz-Verhulst Distribution
}

\author{
M. Ahsanullah ${ }^{1}$, M. Shakil ${ }^{2}$ and B. M. Golam Kibria ${ }^{3}$ \\ ${ }^{1}$ Department of Management Sciences, Rider University, Lawrenceville, NJ 08648, USA \\ ${ }^{2}$ M. Shakil, Department of Mathematics, Miami Dade College \\ Hialeah Campus, Hialeah, Fl 33012, USA \\ ${ }^{3}$ B. M. Golam Kibria, Department of Mathematics and Statistics \\ Florida International University, University Park, Miami, FL 33199, USA \\ ahsan@rider.edu,mshakil@mdc.edu,kibriag@fiu.edu
}

Received 12 February 2013

Accepted 12 December 2013

\begin{abstract}
Characterization of a probability distribution plays an important role in probability and statistics. This paper considers a new characterization of Gompertz-Verhulst distribution. It is hoped that the findings of the paper will be useful for researchers in different fields of applied sciences.
\end{abstract}

Keywords: Characterization, Exponentiated Distribution, Gompertz-Verhulst Distribution.

2010 Mathematics Subject Classifications: 33C15; 60E05; 62E15.

\section{Introduction}

This section discusses Gompertz-Verhulst distribution and some of its properties. 
Gompertz-Verhulst Distribution: A continuous random variable $X$ is said to have GompertzVerhulst (or $\mathrm{G}-\mathrm{V}$ ) distribution if its distribution function $F(x)$ is given by

$$
F(x)=\left(1-\rho e^{-\lambda x}\right)^{\alpha}
$$

for $x>\left(\frac{1}{\lambda}\right) \ln (\rho)>0$, and positive reals $\rho, \lambda$ and $\alpha$. The above model was used by Gompertz and Verhulst to compare known human mortality tables and to represent population growth, see Gompertz [8] and Verhulst [14, 15, and 16]. Further, Ahuja and Nash [2] studied some generalization of Gompertz-Verhulst distribution in their paper, known as the generalized Gompertz-Verhulst distribution, by introducing an additional parameter and made a study of the limiting distributions as the parameters of generalized Gompertz-Verhulst distributions tend to zero or infinity. Besides discussing some properties of the generalized Gompertz-Verhulst distribution, they showed that, by a simple transformation, the Gompertz-Verhulst distribution is related to the Pearson's distributions of types III, VI, and I. For detrails, the interested readers are referred to Ahuja and Nash [2], and references therein. Also, see Ahuja [3], where the distribution of the difference of two independent generalized Gompertz-Verhulst random variables is considered. The probability density function (pdf) $f(x)$ is given by

$$
f(x)=\alpha \rho \lambda e^{-\lambda x}\left(1-\rho e^{-\lambda x}\right)^{\alpha-1} .
$$

The Figure 1.1 gives the pdf's $f(x)$ for $\lambda=1, \alpha=2, \rho=3$ (red), $\lambda=1, \alpha=0.5, \rho=3$ (green), and $\lambda=1, \alpha=2, \rho=4$ (black).

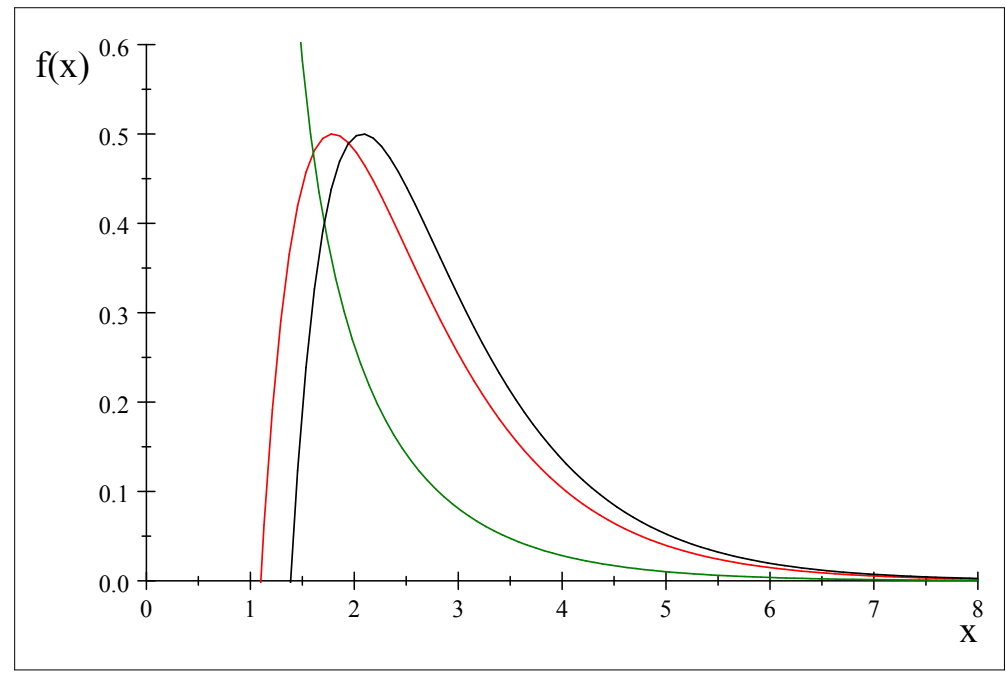

Figure 1.1 PDFs 
The pdf of Gompertz-Verhulst distribution can take different shapes. It is unimodal for $\alpha>1$ and reversed ' $\mathrm{J}$ ' shaped for $\alpha \leq 1$. Further, the density function of Gompertz-Verhulst distribution is $\log$-convex if $\alpha \leq 1$ and $\log$-concave if $\alpha \geq 1$. Also, it has an increasing or decreasing hazard function if $\alpha>1$ or $\alpha<1$ respectively and for $\alpha=1$, the hazard function is constant. The reverse hazard rate $\tau(x)=\frac{f(x)}{F(x)}$ of Gompertz-Verhulst distribution (1.1) is given by

$$
\tau(x)=\frac{\alpha \lambda \rho e^{-\lambda x}}{1-\rho e^{-\lambda x}}
$$

Note that, the exponentiated exponential (EE) distribution, also known as the generalized exponential distribution, as described below, is a particular case of Gompertz-Verhulst distribution (1.1), when $\rho=1$. For distributional properties of the EE distribution, the interested readers are referred to Nadarajah [11], and Nadarajah and Kotz [12], among others.

A random variable $X$ is said to have the EE distribution if its cdf $F_{1}(x)$ is given by

$$
F_{1}(x)=(1-\exp (-\lambda x))^{\alpha}
$$

for $x>0, \lambda>0$ and $\alpha>0$, which is the $\alpha$ th power of the cdf of the standard exponential distribution, which is obviously a particular case of Gompertz-Verhulst distribution (1.1) when $\rho=1$.

The corresponding probability density function (pdf) $f_{1}(x)$ of the EE distribution (1.2) is given by

$$
f_{1}(x)=\alpha \lambda \exp (-\lambda x)[1-\exp (-\lambda x)]^{\alpha-1},
$$

where $\alpha$ and $\lambda$ are the shape and scale parameters respectively. Also, it should be noted that Gompertz-Verhulst distribution (1.1) belongs to the family of $F^{\alpha}$ distributions (see Shakil and Ahsanullah [13]).

Moments and Variance of the Gompertz-Verhulst Distribution: The first and second moments (about the origin) and variance of the random variable $X$ with cdf (1.3) are given below:

First Moment (about the origin): We have

$$
\mu_{1}^{\prime}=E[X]=\int_{\frac{\ln \rho}{\lambda}}^{\infty} x\left(\alpha \rho \lambda \exp (-\lambda x)[1-\rho \exp (-\lambda x)]^{\alpha-1}\right) d x
$$


Taking $u=(1-\rho \exp (-\lambda x))^{\alpha}$, we have

$$
\begin{aligned}
\mu_{1}^{\prime} & =\int_{0}^{1}\left(\frac{\ln \rho}{\lambda}-\frac{1}{\lambda} \ln \left(1-u^{1 / \alpha}\right)\right) d u \\
& =\frac{\ln \rho}{\lambda}-\frac{1}{\lambda} \int_{0}^{1} \ln (1-t) \alpha t^{\alpha-1} d u \\
& =\frac{\ln \rho}{\lambda}+\frac{1}{\lambda}(\psi(\alpha+1)-\psi(1))
\end{aligned}
$$

where $\psi($.$) denotes psi (or digamma) function, see Gradshteyn and Ryzhik [9].$

Second Moment (about the origin): We have

$$
\mu_{2}^{\prime}=E\left[X^{2}\right]=\int_{\frac{\ln \rho}{\lambda}}^{\infty} x^{2}\left(\alpha \rho \lambda \exp (-\lambda x)[1-\rho \exp (-\lambda x)]^{\alpha-1}\right) d x
$$

Taking $u=(1-\rho \exp (-\lambda x))^{\alpha}$, we have

$$
\begin{aligned}
& \mu_{2}^{\prime}= \int_{0}^{1}\left(\frac{\ln \rho}{\lambda}-\frac{1}{\lambda} \ln \left(1-u^{1 / \alpha}\right)\right)^{2} d u \\
&=\left(\frac{\ln \rho}{\lambda}\right)^{2}-\frac{2 \ln \rho}{\lambda^{2}} \int_{0}^{1} \ln (1-t) \alpha t^{\alpha-1} d u \\
&+\frac{1}{\lambda^{2}} \int_{0}^{1}(\ln (1-t))^{2} \alpha t^{\alpha-1} d u \\
&=\left(\frac{\ln \rho}{\lambda}\right)^{2}+\frac{2 \ln \rho}{\lambda^{2}}(\psi(\alpha+1)-\psi(1)) \\
&+\frac{1}{\lambda^{2}}\left[(\psi(\alpha+1)-\psi(1))^{2}+\psi^{\prime}(1)-\psi^{\prime}(\alpha+1)\right]
\end{aligned}
$$


Thus

$$
\operatorname{Var}(\mathrm{X})=\frac{1}{\lambda^{2}}\left(\psi^{\prime}(1)-\psi^{\prime}(\alpha+1)\right)
$$

\section{A Characterization of Gompertz-Verhulst Distribution}

Characterization of a probability distribution plays an important role in probability and statistics. Before a particular probability distribution model is applied to fit the real world data, it is necessary to confirm whether the given probability distribution satisfies the underlying requirements by its characterization. A probability distribution can be characterized through various methods (see, for example, Ahsanullah et al. [1], among others). In recent years, there has been a great interest in the characterizations of probability distributions by truncated moments. For example, the development of the general theory of the characterizations of probability distributions by truncated moment began with the work of Galambos and Kotz [4]. Further development on the characterizations of probability distributions by truncated moments continued with the contributions of many authors and researchers, among them Kotz and Shanbhag [10], Glänzel [5, 6], and Glänzel et al. [7], are notable. However, most of these characterizations are based on a simple relationship between two different moments truncated from the left at the same point. It appears from literature that no attention has been paid on the characterizations of Gompertz-Verhulst distribution by using truncated moment. As pointed out by Glänzel [5], these characterizations may also serve as a basis for parameter estimation.

In this paper, we present a new characterization of Gompertz-Verhulst distribution by using the truncated moment. For this, we have considered a product of reverse hazard rate and another function of the truncated point, as proved in Theorem 2.1 below.

\section{Theorem2.1}

Suppose that $X$ is an absolutely continuous (with respect to Lebesgue measure) random variable with cdf $F(x)$ and pdf $f(x)$. We assume $\beta=\inf \{x \mid F(x)>0\}, \delta=\sup \{x \mid F(x)<1\}, E(X)$ and $f^{\prime}(x)$ exist for all $x \in(\beta, \delta)$. Then if $E(X \mid X \leq x)=g(x) \eta(x)$,

where

$$
\begin{aligned}
& \eta(x)=\frac{f(x)}{F(x)}, \\
& \beta=\frac{\ln \rho}{\lambda}, \text { for positive real numbers } \rho \text { and } \lambda,
\end{aligned}
$$


and

$$
g(x)=\frac{x\left(1-\rho e^{-\lambda x}\right)}{\alpha \rho \lambda e^{-\lambda x}}+\frac{x+\sum_{j=1}^{\alpha}\left(\begin{array}{c}
\alpha \\
j
\end{array}\right)(-\rho)^{j+1} e^{-\lambda j x} \frac{1}{\lambda j}}{\lambda \alpha \rho e^{-\lambda x}\left(1-\rho e^{-\lambda x}\right)^{\alpha-1}}, x \geq \frac{\ln \rho}{\lambda}
$$

for a positive integer $\alpha$ and positive real numbers $\rho$ and $\lambda$ if and only if

$$
f(x)=\alpha \lambda \rho e^{-\lambda x}\left(1-\rho e^{-\lambda x}\right)^{\alpha-1} .
$$

To prove the theorem, we need the following lemma 2.1.

\section{Lemma 2.1}

Suppose that $X$ is an absolutely continuous (with respect to Lebesgue measure) random variable with cdf $F(x)$ and pdf $f(x)$. We assume $\beta=\inf \{x \mid F(x)>0\}, \delta=\sup \{x \mid F(x)<1\}, E(X)$ and $f^{\prime}(x)$ exist for all $x \in(\beta, \delta)$. If $E(X \mid X \leq x)=g(x) \eta(x)$, where $g(x)$ is a differentiable function, and $\eta(x)=\frac{f(x)}{F(x)}$ for all $x>0$, then we have

$$
f(x)=c e^{\int_{\beta}^{x} \frac{u-g^{\prime}(u)}{g(u)} d u},
$$

where $c$ is determined by taking $\int_{\beta}^{\delta} f(x) d x=1$.

\section{Proof of Lemma 2.1}

We have

$$
\frac{\int_{\beta}^{x} u f(u) d u}{F(x)}=\frac{g(x) f(x)}{F(x)}
$$

Thus

$$
\int_{\beta}^{x} u f(u) d u=g(x) f(x) .
$$


Differentiating both sides of the above equation with respect to $x$, we obtain

$$
x f(x)=g^{\prime}(x) f(x)+g(x) f^{\prime}(x) .
$$

On simplification, we get

$$
\frac{f^{\prime}(x)}{f(x)}=\frac{x-g^{\prime}(x)}{g(x)}
$$

Integrating the above equation, we obtain

$$
f(x)=c e^{\int_{\beta}^{x} \frac{u-g^{\prime}(u)}{g(u)} d u},
$$

where $c$ is determined such that

$$
\int_{\beta}^{\delta} f(x) d x=1
$$

\section{Proof of Theorem 2.1}

Suppose

$$
f(x)=\alpha \lambda \rho e^{-\lambda x}\left(1-\rho e^{-\lambda x}\right)^{\alpha-1}, x \geq 0 .
$$

Then it is easy to show that

$$
E(X \mid X \leq x)=\frac{x\left(1-\rho e^{-\lambda x}\right)}{\alpha \rho \lambda e^{-\lambda x}}+\frac{x+\sum_{j=1}^{\alpha}\left(\begin{array}{l}
\alpha \\
j
\end{array}\right)(-\rho)^{j+1} e^{-\lambda j x} \frac{1}{\lambda j}}{\lambda \alpha \rho e^{-\lambda x}\left(1-\rho e^{-\lambda x}\right)^{\alpha-1}} .
$$

We will prove here the only if condition.

Suppose that

$$
g(x)=\frac{x\left(1-\rho e^{-\lambda x}\right)}{\alpha \rho \lambda e^{-\lambda x}}+\frac{x+\sum_{j=1}^{\alpha}\left(\begin{array}{c}
\alpha \\
j
\end{array}\right)(-\rho)^{j+1} e^{-\lambda j x} \frac{1}{\lambda j}}{\lambda \alpha \rho e^{-\lambda x}\left(1-\rho e^{-\lambda x}\right)^{\alpha-1}},
$$


then

$$
g^{\prime}(x)=x+\left[\frac{x\left(1-\rho e^{-\lambda x}\right)^{\alpha}}{\alpha \rho \lambda e^{-\lambda x}\left(1-\rho e^{-\lambda x}\right)^{\alpha-1}}+\frac{x+\sum_{j=1}^{\alpha}\left(\begin{array}{c}
\alpha \\
j
\end{array}\right)(-\rho)^{j+1} e^{-\lambda j x} \frac{1}{\lambda j}}{\lambda \alpha \rho e^{-\lambda x}\left(1-\rho e^{-\lambda x}\right)^{\alpha-1}}\right] H(x),
$$

where

$$
H(x)=-\lambda+\frac{(\alpha-1) \lambda \rho e^{-\lambda x}}{1-\rho e^{-\lambda x}}
$$

Thus

$$
\frac{f^{\prime}(x)}{f(x)}=\frac{x-g^{\prime}(x)}{g(x)}=H(x)=-\lambda+\frac{(\alpha-1) \lambda \rho e^{-\lambda x}}{1-\rho e^{-\lambda x}} .
$$

On integrating the above equation, we

$$
f(x)=c e^{\int_{\frac{\ln \rho}{\lambda}}^{x} H(u) d u}
$$

Now

$$
\begin{aligned}
\int_{\frac{\ln \rho}{\lambda}}^{x} H(u) d u & =\int_{\frac{\ln \rho}{\lambda}}^{x}\left(-\lambda+\frac{(\alpha-1) \lambda \rho e^{-\lambda u}}{1-\rho e^{-\lambda u}}\right) d u \\
& =-\lambda\left(x-\frac{\ln \rho}{\lambda}\right)+(\alpha-1) \ln \left(1-\rho e^{-\lambda u}\right) .
\end{aligned}
$$

Thus

$$
f(x)=c \rho e^{-\lambda x}\left(1-\rho e^{-\lambda x}\right)^{\alpha-1},
$$

where

$$
\frac{1}{c}=\int_{\frac{\ln \rho}{\lambda}}^{x} e^{-\lambda x}\left(1-\rho e^{-\lambda x}\right)^{\alpha-1} d x=\frac{1}{\alpha \lambda \rho} .
$$

This completes the proof of Theorem 2.1. 


\section{Concluding Remarks}

Characterization of a probability distribution plays an important role in probability and statistics. Before a particular probability distribution model is applied to fit the real world data, it is necessary to confirm whether the given probability distribution satisfies the underlying requirements by its characterization. A probability distribution can be characterized through various methods. In this paper, a new characterization of Gompertz-Verhulst distribution has been established. For this, we have considered a product of reverse hazard rate and another function of the truncated point. For the sake of completeness, some distributional properties of Gompertz-Verhulst distribution have also been discussed. It is hoped that the findings of this paper will be useful for the practitioners in various fields of studies and further enhancement of research in the field of distribution theory and its applications.

\section{Acknowledgment}

The authors would like to thank the referees and the editor for helpful suggestions which improved the quality and presentation of the paper.

\section{References}

[1] Ahsanullah, M., Kibria, B. M. G. and Shakil, M. (2014). Normal and Student's t Distributions and Their Applications. Atlantis Press, Paris, France.

[2] Ahuja, J. C., and Nash, S. W. (1967). The generalized Gompertz-Verhulst family of distributions. Sankhya, Ser. A., 29, 141-156.

[3] Ahuja, J. C. (1969). On certain properties of the generalized Gompertz distribution. Sankhyā: The Indian Journal of Statistics, Series B, 31, 541-544.

[4] Galambos, J., and Kotz, S. (1978). Characterizations of probability distributions. A unified approach with an emphasis on exponential and related models, Lecture Notes in Mathematics, 675, Springer, Berlin, Germany.

[5] Glänzel, W. (1987). A characterization theorem based on truncated moments and its application to some distribution families, Mathematical Statistics and Probability Theory (Bad Tatzmannsdorf, 1986), Vol. B, 75 - 84, Reidel, Dordrecht, Germany.

[6] Glänzel, W. (1990). Some consequences of a characterization theorem based on truncated moments, Statistics, 21, 613-618.

[7] Glänzel, W., Telcs, A. and Schubert, A. (1984) Characterization by truncated moments and its application to Pearson-type distributions, Z. Wahrsch. Verw. Gebiete, 66, 173-183. [8] Gompertz, B. (1825). On the nature of the function expressive of the law of human mortality, and on a new mode of determining the value of life contingencies. Philosophical Transactions of the Royal Society London, 115, 513-585. 
[9] Gradshteyn, I. S., and Ryzhik, I. M. (1980). Table of Integrals, Series, and Products (6th edition). Academic Press, San Diego.

[10] Kotz, S. and Shanbhag, D.N. (1980). Some new approaches to probability distributions. Advances in Applied Probability, 12, 903-921

[11] Nadarajah, S. (2011). The exponentiated exponential distribution: a survey. AStA Advances in Statistical Analysis, 95, 3, 219-251.

[12] Nadarajah, S., and Kotz, S. (2006). The exponentiated type distributions. Acta Applicandae Mathematicae, 92, 97-111.

[13] Shakil, M. and Ahsanullah, M. (2012). Review on order statistics and record values from $F^{\alpha}$ distributions, Pak. J. Stat. Oper. Res., VIII, 101-120.

[14] Verhulst, P. F. (1838). Notice sur la loi la population suit dans son accroissement", Correspondence mathematique et physique, publiee L. A. J. Quetelet, 10, 113-121.

[15] Verhulst, P. F. (1845). Recherches mathematiques sur la loi-d'-accroissement de la population", Nouvelles Memoires de l'Academie Royale des Sciencs et Belles-Lettres de Bruxelles [i.e., Memoires, Series 2], 18, 38 pp.

[16] Verhulst, P. F. (1847). Deuxieme memoire sur la loi d'accroissement de la population", Memoires de l'Academie Royale des Sciences, des Lettres et des Beaux-Arts de Belgique, Series 2, 20, $32 \mathrm{pp}$. 